\title{
Study on the Qixi Literature Research of the Han and Wei Six Dynasties
}

\author{
Ruifang Zhang \\ Weinan Normal University, College of Humanities, Shaanxi, Weinan, 714099 \\ Southeast University, School of Arts, Jiangsu, Nanjing 210096
}

Keywords: Han and Wei Six Dynasties; Qixi Literature; poetic imagery; cultural connotation

\begin{abstract}
Qixi literature refers to the poetry of Chinese classical literature with the theme of Tanabata or the use of the image of the Cowherd and the Weaver Girl. The Tanabata originated from the celestial body worship. The myths and legends of the Cowherd and the Weaver Girl touched the literati, which produced a large number of Qixi literature. It has become a literary theme with a unique national cultural color in the history of Chinese literature. The poet framed the love story of the Cowherd and the Weaver Girl, and the firm and persistent love story was constructed through words. Especially in the Han and Wei Six Dynasties, the social turmoil and the war were frequent. The literary works of this period were mainly full with pain, sadness and jealousy. The tragic story of the Cowherd and the Weaver Girl in the legend of Qixi is also the projection of the author's own experience and sigh. Therefore, under his own emotional control, the writer has created a seven-day literary work with rich connotations and beautiful charm. The paper mainly discusses the cultural connotation of Zhongqixi in the Han, Wei and Six Dynasties, and interprets the text through the image analysis of Qixi literature.
\end{abstract}

\section{The Cultural Connotation of Qixi}

Qixi Festival is the seventh day of the seventh lunar month in China. It has a direct relationship with the ancient Chinese folk time concept. The ancients believed that the seven were positive numbers, and the yang numbers overlap on July 7th, which is a special day to be commemorated; and the lunar calendar is a bumper harvest in July. It can be said that the Qixi Festival has been endowed with rich cultural connotations since its inception. Weavers have the six divine natures of the Lord's fruit, the main livelihood, the main wealth, the birth of the child, the shelter of the child, the main life, and the main marriage. Specifically, the cultural connotation of Tanabata is mainly reflected in three aspects: farming culture, unshakable love concept and heavy birth. The phenomenon of patriarchalism in farming society is very common. Women's social status is low, and marriage is an important factor in determining their destiny. Therefore, women in ancient society have to learn various female red skills from an early age to meet the requirements of a qualified wife. The main target of Tanabata is women, so the woman will hope that she will be smart and intelligent. The concept of love expressed by Tanabata is an embodiment of its important cultural connotations. In ancient times, men and women were so poor that many women became victims of marriage, and they longed for a happy marriage. Tanabata uses the love story of the Cowherd and the Weaver Girl as the carrier. Chinese classical novels, operas and folk art all advocate the reunion ending. The Tanabata meeting is also the subtlety of the story of the Cowherd and the Weaver Girl, although the two loved ones cannot pilgrimage, but Every year, July 7th can meet, and the Cowherd and the Weaver Girl are full of expectations in the torment, which is in line with the traditional Chinese concept of love. In addition, for the ancients, fertility is also a mysterious thing, and many folk legends are the content of the gods. In ancient times, due to medical conditions, the survival rate of children after birth was low, so the worship of the goddess would be considered to protect the mother and child. The Cowherd and the Weaver Girl have a son and a daughter. For the Chinese, they are made into a "good" character. This kind of enjoyment of happiness is the most proud thing in life, so the blind man becomes an important holiday custom of Qixi Festival. 


\section{The Image of "Moon" in Qixi Literature of Han and Wei Six Dynasties}

Since the Tanabata and the Weaver Girl's Tanabata meeting has a close relationship with the moon, the "month" in the Qixi literature of the Han, Wei and Six Dynasties has the highest frequency of occurrence, and the literary works often include the words of the moon, the moon, the moon, the string and the moon. The ancients believed that the sun was positive, representing the male, the moon was negative, representing the female, and the Tanabata literature was mostly based on the female perspective. Therefore, the "month" became an important element of the artistic conception of the poetry, expressing the author's delicateness through various descriptions of the month. Most of the "months" described in the Qixi literature of the Han, Wei and Six Dynasties express the sentiment of the Cowherd and the Weaver Girl in the next period of the Moonlight. It is not only the literature of the Han and Wei Dynasties, but also the specific image of the moon image in the literary works of the past dynasties. Reunion, and send acacia through the moon, or through the moon. When verses depicting the moon appear in literary works, involuntary people will make feelings of thoughts and sorrow through the moon, especially the special theme of Tanabata. Tanabata itself comes from the tragic story of the Cowherd and the Weaver Girl, and the Cowherd and the Weaver Girl are in the moon. The details of the story of the meeting, the well-behaved folk activities of the moon, and the fact that the moon itself is negative, have resulted in a large number of images of the moon in the Qixi literature. For example, "Mingyue illuminates my bed, Xinghan Xiliu night is not central" (Cao Wei, "Yan Ge Xing"), " the night fog is white" (Bao Zhao, "And Wang Yixing Qixi Poetry"), poetry The clear moonlight reflects the loneliness and sadness of the wanderer.

\section{The "Autumn" Image in Qixi Literature of Han and Wei Six Dynasties}

In autumn, everything begins to wither, giving people a feeling of sadness. Therefore, "sorrowful autumn" in literary works is an important theme, and autumn image has become a prototype image in literary works. The Qixi literature of the Han, Wei and Six Dynasties was expressed through the characteristic objects in the description of the autumn imagery, such as "autumn wind", "autumn cloud", "autumn night", etc., no matter what kind of object gives a cold, sad feel. Qixi Festival is in the early autumn period, so the Mid-Autumn Festival image of the Han and Wei Dynasties and Qixi literature has obvious time hints.

The autumn imagery of Qixi literature in the Han, Wei and Six Dynasties can be divided into the following categories: First, the desolation and depression of autumn. For example, "the autumn wind is bleak and the weather is cool, the grass is shaken and exposed as frost" (Cao Wei, "Yan Ge Xing"), "Bai Lu Yue Xia, the autumn wind branch is fresh" (Xiao Yan, "Qi Xi Shi"), "Autumn Wind", "Lu is a cream", "Bailu" and so on is a description of the phenomenon that the weather is getting colder and the cold dew is getting heavier in autumn, and the arrival of autumn means that more than half of the year has passed, and the autumn time is very short, so it is easy to make people associate To the time is easy to pass. Second, autumn is the limit of time. The Cowherd and the Weaver Girl can only meet once a year, and seeing each other is the beginning of separation. The Cowherd and the Weaver Girl have both hope and joy in the process of waiting for each other, and they have the reluctance to leave. There is helplessness for the long-term waiting, and this time of seeing and leaving is in the fall, so there is the following verse: "The period of the cows and the weavers in the autumn, the mountains and the waters are infinite" (Fu Xuan, "The Four Poems 》), "Three Springs mourn and cry, Jiuqiu Xin song" (Qingshang Qufu, "Seven Nights Girl Songs Group"), "After the autumn, a photo, a photo of the dust" (Shen, "Weaver Give the cowboy poems) and so on. Finally, describe the scenes of the Cowherd and the Weaver Girl's autumn meeting, such as "Stop the Spring Festival, stop the box and move the Autumn" (Xie Zhuang, "The Seven-Year Night Yak Women Should Make Poems"), "Autumn Clouds, Clouds, Stops "(Xing Wei, "The Seventh Festival Poems"), "Han Qu Tian Tian Leng, Riverside Laurel Autumn" (Jiang Zong, "Qi Xi Po") and so on. When the "autumn period" arrived, the weavers "stopped the color" and the cowherd also "stopped the box and moved the autumn", all in order to go to the meeting. The two 
people attach great importance to the gathering once a year, which also expresses the cowherd from the side. The difficulty of meeting with the Weaver Girl, it can be seen that the "autumn meeting" shows the painful feelings of the Cowherd and the Weaver Girl who can't get together because of the Han Han barrier.

Because the time of the meeting between the Cowherd and the Weaver Girl has a common point of convergence with the theme of "Sorrowful Autumn" in Chinese literary works, there are a lot of autumn images in the Qixi literature of the Han, Wei and Six Dynasties, and there is a sense of sorrow and coldness in the lines between the lines. The tragedy of love is vividly revealed.

\section{The Image of Hehan in Qixi Literature of Han and Wei Six Dynasties}

The barrier of Hehan is the main factor that the Cowherd and the Weaver Girl can't meet. In the Qixi literature of the Han and Wei Dynasties, Hehan also called it "Xinghe", "Changhe", "Yunhan", "Tianhe", "Tianhuang", etc., and the image of Hehan can be divided into The following are the following: First, symbolize the distant distance. The most typical one is the "Nine-Nine-Nine" in the "Nineteen Ancient Poems": The distance between the shore and the other shore, "Ying Ying", " outlines a river with a glimmer of light, separating the Cowherd and the Weaver Girl, although the river is light and bright, but However, because the two people are "not fluent", they are particularly embarrassing, making people full of sadness. Secondly, He Han was also given a layer of tragic meaning, expressing the author's strong and lasting feelings through the bitter and grievances that the Cowherd and the Weavers could not meet each other. For example, "Zhao Zhao Qing Han Hui, Shuguang Tianbu, the Niubei back, the female southeast Gu" ( $\mathrm{Lu} \mathrm{Ji}$, "Pseudo Lancôme"), the Cowherd and the Weaver Girl from the bright river Han, respectively, Niubei back, weaving female southeast Gu," the two turned back to look forward to, Yiyi farewell, bitter helpless, and reluctant to care about it, set off "Zhao Zhao Qing Han Hui", but also added a bitterness and sorrow. He Han not only blocked the meeting of the Cowherd and the Weaver, but also the place where the Cowherd and the Weaver Girl met, so Hehan became a perfect place to sit on Acacia. Finally, He Han is still a symbol of hope. As mentioned above, although He Han blocked the meeting between the Cowherd and the Weaver Girl, the meeting place of the two was also here, so it gave the Cowherd and the Weaver a glimmer of hope. For example, "Who is Yun Changhe Yao, quite a play promotes Yue Yue" (Liu Wei, "The Seventh Eve Cattle Poetry"), these two sentences show that the Cowherd and the Weaver Girl are very eager to go to the party, so that the pain of daily waiting is The happiness of the gathering is diluted. In the deeper interpretation, the Han and Han dynasties in the Qixi literature of the Han, Wei and Six Dynasties also symbolize the passage of time and the cycle of time. Water itself has the meaning of time lapse. Although He Han is a galaxy composed of stars, it can also be transformed into a river of water flow. Therefore, the image of He Han also expresses that the Cowherd and the Weaver Girl can only wait in the middle of the time, only in Han In the soup of water, the love tragedy of the people.

\section{The Image of the Weaver Girl in Qixi Literature of Han and Wei Six Dynasties}

The Weaver Girl is a combination of truth, goodness and beauty in the Qixi literature of the Han and Wei Dynasties. Almost all literary works describe it as a supple, stubborn, firm, hardworking, intelligent image. Treating love is patient, firm, pure and sincere, and later generations are She was deeply moved by her. While analyzing historical reasons, the shaping of the image of the Weaver Girl is not unrelated to the prevalence of the poetry and poetry of the Wei, Jin, Southern and Northern Dynasties, although the love of the Cowherd is positive, but this kind of initiative "sends love, ends with ritual”. In line with Confucian ethics, it is accepted and praised by the majority of literati. Therefore, the prototype of the Weaver Girl can be regarded as an extension of the image of the Gaotang Goddess and Luo Shen, which were secularized by feudal ethics. For example, "Yang Yang Yun in Jing Meng, Fu Luo in Chen Xiang" (Xie Chou, "Qi Xi Fu"), Yang Yun Jing Meng is Gao Tang Goddess, Luo Shi Chen Xiang refers to Luo Shui; The sidecar is stationed in the Seven Miles, and the phoenix drives out the opening... It is faintly like Luolu, and it seems like Gaotang” 
(He Xun, "The Seventh Festival Poems") also believes that the Weaver Girl is similar to the Gaotang Goddess and Luo Shen. They not only have beautiful looks. Superman's divine power, and there is also a brave pursuit of love, loyal heart. In the Qixi literature of the Han, Wei and Six Dynasties, in addition to describing the love and thoughts of the Weaver Girl on the Cowherd, the psychological and graceful dress of the Weaver Girl when she went to the meeting was also described by the literati. For example, "Six Long Fen Yao Yao, Wen Yu negative Qiong car, Huo Dan Binghua candle, Su female holding Qionghua, fiber flag if spit electricity, Zhu Gai Ruo Xia" (Wang Jian, "Qi Xi Guan Weaving Female Poetry") can be seen in the Weaver Girl When I went to the meeting, the scene was very grand and gorgeous. "Six Dragons", "Qiong", "Qiong Hua", "Zhu Gai", "Fiber Flag", etc., both described the dressing of the Weaver Girl and reflected its grace and elegance. The dressing of the Weaver Girl just shows her attention to the cowherd, which reflects her positive and active side in her love with the Cowherd.

In short, the Qixi literature of the Han, Wei and Six Dynasties laid the emotional tone of the later generations of Qixi literature. Later generations of Qixi literature have learned from the Han, Wei and Six Dynasties Qixi literature, and their emotional themes are mostly based on lovesickness and suffering. Judging from the development of Chinese classical poetry, Qixi literature has a rich cultural connotation as a unique creative subject, so it has a high literary value and artistic charm.

\section{References}

[1] Zhao Yufu. On the formation of the plot of Wuhuan Bridge in the story of Niu Niu and its aesthetic significance. Beijing Social Science [J].1990(1):374-375

[2] Zhao Yufu. On the emergence and theme of the story of Cowherd and Weaver Girl. Journal of Northwest Normal University [J].1990(4):636-637

[3] Li Honglin. A Textual Research on the Hanshui and Tianhan Culture-On the Origin of the Myths of the Cowherd and the Weaver Girl[J].Wu Dang Academic Journal[J].1993(4):126-127

[4] Du Hanhua, Wang Bitao, Yu Haipeng. A Study on the Origin of the "The Cowherd and the Weaver Girl" and "The Qixi Festival"[J]. Journal of Xiangfan Vocational and Technical College. 2004(5): 976-977

[5]Zhou Shufang. Qixi Poetry: The Flame of Natural Burning and Warm Poet's Soul [J].Journal of Yanbian University.2002 (5):1095-1096

[6] Li Ting. The Evolution of Qixi Literature in the Han, Wei and Six Dynasties [J]. Journal of Shanghai Jiaotong University. 2003(2): 164

[7] Hong Shuhua. Seeing the Prototype Characteristics of Chinese Classical Poetry from the Imagery of "The Cowherd and the Weaver Girl". Jianghan Forum [J].2003(5):284-285 